\title{
Advanced prostate cancer experimental radioactive treatment-clinical trial decision making: patient experiences
}

\author{
Bianca Viljoen (1) , ${ }^{1}$ Michael S Hofman, ${ }^{2,3}$ Suzanne K Chambers, ${ }^{4,5,6,7,8}$ \\ Jeff Dunn, ${ }^{6,7}$ Haryana Dhillon (1) ${ }^{9}$ Ian D Davis, ${ }^{10,11}$ Nicholas Ralph ${ }^{1,4,6}$
}

- Additional supplemental material is published online only. To view, please visit the journal online (http://dx.doi. org/10.1136/bmispcare-2021 002994).

For numbered affiliations see end of article.

\section{Correspondence to}

Associate Professor Nicholas Ralph, School of Nursing and Midwifery, University of Southern Queensland, Ipswich, Australia; nicholas.ralph@usq.edu.au

Received 16 February 2021 Accepted 8 July 2021

\section{Check for updates}

(c) Author(s) (or their employer(s)) 2021. No commercial re-use. See rights and permissions. Published by BMJ.

\footnotetext{
To cite: Viljoen $B$, Hofman MS, Chambers SK, et al. BMJ Supportive \& Palliative Care Epub ahead of print: [please include Day Month Year]. doi:10.1136/ bmispcare-2021-002994
}

\section{ABSTRACT}

Objectives Nested qualitative studies within clinical trials provide the opportunity to better understand participant experiences of participation and identify areas where improved support is required. The purpose of this qualitative study is to describe the lived experiences of men with advanced prostate cancer participating in the TheraP trial; a randomised trial of ${ }^{177}$ Lu-PSMA-617 compared with cabazitaxel chemotherapy.

Methods Fifteen men with advanced prostate cancer were recruited from the TheraP clinical trial and interviewed at three time points during the trial. Interviews were inductively analysed using thematic analysis. This research paper reports the results from the baseline interview at commencement of the trial, focusing specifically on participants' enrolment experiences.

Results Four themes were identified representing the lived experiences of men with advanced prostate cancer deciding to participate in the TheraP trial: (1) hoping to survive; (2) needing to feel informed; (3) choosing to participate and (4) being randomised. The process of deciding to enrol in a clinical trial is filled with indecision, emotional difficulties and focused on a desire to live.

Conclusions For men with advanced prostate cancer, the experience of deciding to enrol in a clinical trial is principally driven by a desire to survive but interlinked with the need to make an informed decision as participants in this study expressed a preference for allocation to the experimental arm. Men seeking to enrol in clinical trials of new prostate cancer treatments would benefit from improved informational and decision support.

Trial registration number NCT03392428, ANZUP1603.

\section{Key messages}

What was already known?

- Clinical trials are an increasingly soughtafter source of curative treatment for people with advanced cancer.

- There are gaps in the literature around how best to support people participating in trial who have advanced cancer.

What are the new findings?

- The experience of trial participation for men with advanced prostate cancer can be represented by 'hoping to survive', 'needing to feel informed', 'choosing to participate' and 'being randomised'.

- The process of deciding to enrol in a clinical trial if filled with indecision, emotional difficulties and focused on a desire to live.

What is their significance?

a. Clinical: This is the first study to articulate the experience of men with advanced prostate cancer who participate in a trial.

b. Research: Implications from this study highlight the need for tailored information and improved psychological support interventions for men with advanced prostate cancer.

\section{BACKGROUND}

Prostate cancer is the second most frequently diagnosed cancer and the sixth foremost cause of cancer death among men worldwide. ${ }^{1}$ Globally, an estimated 358000 men died from the disease in $2018 .^{2}$ Those living with advanced disease experience poor quality of life (QOL), greater psychological distress and unmet supportive care needs compared with men with localised disease. ${ }^{3}{ }^{4}$ Men with advanced disease report high physical and psychosocial burdens from cancer and 
treatment side effects including pain, sarcopenia, deteriorating bone health, cognitive and sexual dysfunction, fatigue, hot flushes, urinary incontinence, bowel changes, increased fat mass and body image alterations and psychosocial distress. ${ }^{5-10}$ It is essential new treatments extend life without increasing health-related burdens for men with advanced prostate cancer.

The search for new effective and acceptable treatments is of interest to cancer survivors the world over amidst a significant uplift in global trial registrations from 1255 in 2000 to 348177 studies in $2020 .^{11}$ As of August 2020, a record 52347 trials were registered actively recruiting participants ${ }^{11}$ yet barriers to participation have continued. Although around $70 \%$ of people living with cancer are willing to participate in clinical trials, ${ }^{12}$ research suggests fewer than $5 \%$ ultimately enrol. ${ }^{13} 14$

For individuals with advanced prostate cancer, the opportunity to enrol in a clinical trial could provide hope, ${ }^{15,} 16$ specialist care ${ }^{15}$ and improve health. ${ }^{17}$ TheraP is an Australian multisite randomised phase II, non-blinded trial of ${ }^{177}$ Lu-PSMA-617 (Lu-PSMA) theranostics versus cabazitaxel chemotherapy, a standard treatment, in men with progressive metastatic castration resistance prostate cancer. ${ }^{18}$ Lu-PSMA is a radiolabelled small molecule that binds to prostatespecific membrane antigen (PSMA) enabling delivery of targeted radiation to sites of prostate cancer metastases. ${ }^{19,20}$ Results showed that Lu-PSMA-617 is more active with higher PSA responses, more radiographic responses and longer progression-free survival. ${ }^{21}$ It also demonstrated less adverse events and improvements in some patient-reported outcome domains.

With individuals seeking clinical trials as a potential source of curative treatment, it is necessary to understand the experience and supportive needs of patients deciding to participate in a cancer trial. ${ }^{22}$ Embedding qualitative studies within clinical trials have been recognised as highly effective in influencing clinical trials design ${ }^{23}$ and has the potential to improve recruitment, retention and trial conduct, assist trialists sensitivity towards participants and guide researchers towards implementing more effective interventions in future trials, however it is infrequently used. ${ }^{24}$ The use of qualitative research may enable better understanding of the holistic illness experience of men with progressive cancer. ${ }^{25}$

Few clinical trials have nested qualitative studies as a mechanism for exploring the experience of participants in trials of advanced cancer treatments and none in men with advanced prostate cancer deciding to enrol and participate in a clinical trial. Accordingly, we undertook a qualitative study describing the lived experiences of men with advanced prostate cancer enrolled in the TheraP clinical trial. In this paper, we report participant motivations, perspectives and experiences of deciding to enrol in the TheraP trial.

\section{METHODS}

\section{Aim and objectives}

The aim of this nested qualitative study is to describe the lived experiences of men with advanced prostate cancer participating in the TheraP trial; a randomised trial of ${ }^{177} \mathrm{Lu}$-PSMA-617 compared with cabazitaxel chemotherapy. It is anticipated that the unique context of clinical trial enrolment among men with advanced prostate cancer elicits similarly unique needs additional to those already required by these men. To this end, the objective of this study is to identify and describe the lived experience of deciding to enrol in a clinical trial as well as the factors that inform the decision to enrol in a clinical trial among men with advanced prostate cancer.

\section{Broad eligibility criteria for TheraP clinical trial}

Men with metastatic castration resistant prostate cancer considered to receive cabazitaxel as the following applicable standard treatment were recruited for the TheraP clinical trial. Participants required a performance status of $0-2$ according to the Eastern Cooperative Oncology Group and sufficient haematological, renal and liver activity. Previous treatment with androgen receptor-directed therapy was permitted. PET-CT scans including gallium-68 $\left[{ }^{68} \mathrm{Ga}\right] \mathrm{Ga}-\mathrm{PSMA}-11$ and 2-flourine-18 $\left[{ }^{18} \mathrm{~F}\right]$ fluoro-2deoxy-D-glucose (FDG) were completed in which the eligibility criteria included PSMA-positive disease and no sites of metastatic disease with conflicting FDGpositive and PSMA-negative findings. ${ }^{26}$

\section{Participants}

Fifteen men with advanced prostate cancer were recruited from Peter MacCallum Cancer Centre (Melbourne) and Royal Brisbane and Women's Hospital (Brisbane) participating in the TheraP clinical trial of ${ }^{177}$ Lu-PSMA versus cabazitaxel. ${ }^{21}$ In addition to criteria for TheraP trial recruitment, participants in QualTheraP were required to: (1) read and speak English; (2) have no history of head injury, dementia or psychiatric illness and (3) have phone access.

\section{Procedure}

Eligible participants were approached by trial clinicians and provided with an information brochure about the QualTheraP substudy and a 'permission to contact' form. The invitation process was designed to explain the research in more detail, assess the participants interest in participating, determine eligibility and make an appointment to commence the interview process. Recruitment to the QualTheraP study commenced 3 April 2019 and ceased 7 January 2020.

All patients were interviewed via telephone, the most convenient form of communication for participants. All participants provided written informed consent. Additional verbal consent was confirmed at the commencement of each interview. Interviews 


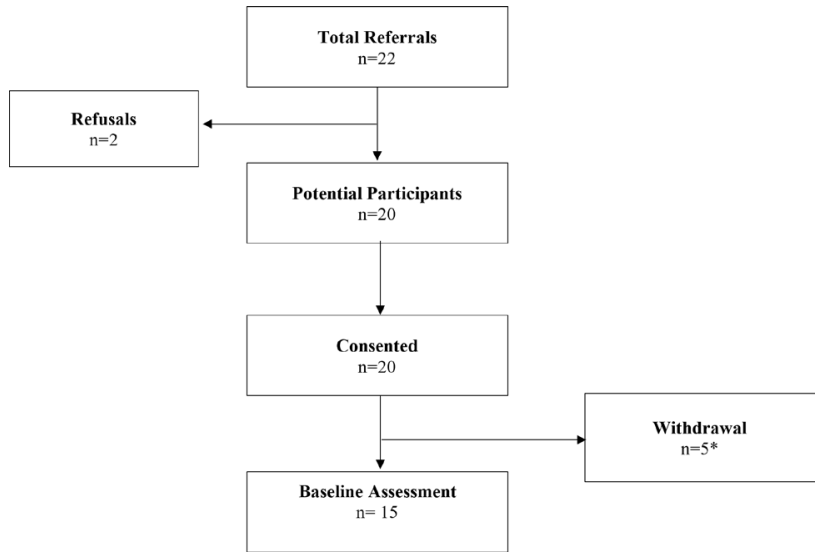

Figure 1 Study flow diagram. * Reasons for withdrawal include failing kidneys $(n=1)$, the mutual agreement between physician and patient not to continue $(n=1)$, a desire to end treatment and be placed into palliative care $(n=2)$ and death from unrelated disease $(n=1)$ and aortic aneurysm $(n=1)$.

were scheduled prospectively at three time points: after enrolment but prerandomisation as a baseline interview (commencement of the trial), midpoint of treatment at 11 weeks and completion of treatment at 23 weeks, with an additional follow-up interview, to explore longitudinal experiences of the main TheraP trial.

Interviews at prerandomisation were carried out for this study by two researchers (BV, NR), who were previously unknown to participants. An interview protocol was used (online supplemental table 1), which covered motivations for trial enrolment, understanding of trial processes and supportive care needs. The protocol, study research questions and qualitative semi-structured interview method, encouraged participants to speak freely and cultivate their own narratives about the trial journey more broadly. Interviews lasted approximately 1 hour. The interviews were audiorecorded, transcribed verbatim and anonymised. This research paper reports results from the baseline interview at commencement of the trial. The final number of recruited participants for the initial assessment is shown in figure 1. The participants' demographic data are shown in online supplemental table 2.

\section{Data analysis}

Following this methodology, thematic analysis was performed with results presented as narrative synthesis. The transcripts of interviews were coded and categorised (BV). Researchers (BV, NR) analysed the transcripts for common themes of trial participants' experiences. Coders used the approach to thematic analysis by Braun and Clarke ${ }^{27}$ and a preliminary coding schema was created in NVIVO V.12 (QSR International 2018) by coding all transcripts with two additional members of the study team reviewing theme categorisation to improve the reliability and validity of the data. Reporting of data conforms with the Criteria for Reporting Qualitative Studies guidelines. ${ }^{28}$

\section{RESUITS}

Four themes were identified representing the lived experiences of men with advanced prostate cancer deciding to participate in the TheraP trial: (1) hoping to survive; (2) needing to feel informed; (3) choosing to participate and (4) being randomised. Selected quotations are shown in online supplemental table 3 to represent the identified themes and participants' perspectives.

\section{Hoping to survive}

Participants reported becoming aware of the TheraP trial in the context of their advancing cancer, extensive side effects from cancer and related treatment, desire to find an effective treatment for their disease and to live. Amidst a variety of reported treatments such as hormone therapy, chemotherapy, radiation therapy and radical prostatectomy, participants reported a sense of deteriorating health as the burden from symptoms such as pain, fatigue, urinary incontinence, haematuria, loss of appetite and weight and sexual dysfunction worsened. Sensing their deterioration, participants experienced psychosocial burdens such as stress, anxiety, uncertainty and fear in addition to a feeling of hopelessness that previous treatments had failed to arrest their disease and left them with debilitating side effects such as urinary incontinence and haematuria. Most described being told by their oncologist that due to their disease advancing, there were only limited treatment options available to them. Participants described their desire to live and urgently seeking alternative treatment to extend and improve their QOL. In the context of limited treatment options, participants expressed newfound hope of obtaining benefit from improving, extending or saving their life by receiving Lu-PSMA:

\begin{abstract}
So, we are just going to run out of treatment to keep me going you know. At some point all of this stuff we've got is going to stop working and you know, we are going to hit end of the road sort of thing. (ID011) I'm on the lookout for, and hopeful that there might be various things which can extend it (life). (ID004)
\end{abstract}

\section{Needing to feel informed}

Contemporaneous to participants' hope for improved survival was their search for information on available treatment options and the likelihood of these being lifeextending for them or reducing debilitating symptoms. Participants referred to using a variety of mechanisms to source the best information about new treatments such as internet search engines, forums and support groups to identify possible treatment options in clinical trials and take this information to their treating oncologist for discussion. Many reported being aware 
of the experimental Lu-PSMA treatment and expressed optimism and hope about its effectiveness.

What interested me was the fact that it seemed to be giving the best results from anything that we'd heard about. We'd more often spoken to people who'd been on it both overseas and here who'd had it and it extended their life by some period of time. And so-and that was, of course, the ultimate gameultimate aim, I guess, of any of these things that you're on... whatever it is, is to provide a satisfactory sort extension of your life, I guess. (ID006)

The interaction between patient and physician was essential to resolving their uncertainty by evaluating whether trial treatments might be effective for their disease and to gain access to trials.

He (oncologist) said look maybe we can look at a trial that might attack it in a different way and give your body a bit of a chance to recover more'. That really interested me because I was really struggling so then he sat me down and gave me some information about it. (ID011)

During trial recruitment, participants reported difficulty in comprehending trial information and processes as written materials were complex and voluminous. As a mechanism for understanding the trial they were enrolled in, participants reported searching the internet to help comprehend the written materials they received, and the verbal information communicated to them. Participants also expressed their preference for more succinct trial information including the trial aim, side effects of treatment, routes of therapeutic administration and an estimate of the duration of treatment effectiveness for their disease.

So, if it just comes back to the normal man's sort of language, I think it would be a lot easier for a lot of people to understand. I understand a lot of these chemotherapies and that have long names and all of the bits and pieces to it, but if they could explain it a little bit simpler. (ID003)

I don't like getting too much information. I just want the bare bones and that's really... so for me, that was enough. It told me the side effects, it told me the possible routes, it told, and you know, it won't hold the cancer for huge amounts of periods, it will only be sort of up to 12 to 18 months at the most. (ID011)

Participants commonly reported difficulty relaying information received from treating health professionals and trialists relevant to their family members. Consequently, participants sourced further information about the trial with their family members, kept their own records, recorded consultations and obtained further information from support groups on social media.

I did research more, but I thought the notes regarding the trial were very comprehensive and yeah, they were good, they gave me a, certainly a good understanding, but then, as I say, I did read up about the different treatments and, you know, did a bit more research so, I had a bit more thorough information, certainly, certainly the trial documentation was well presented. (ID014)

\section{Choosing to participate}

Participants reported a range of motivations they felt were key determinants in choosing whether to participate in the TheraP trial, including personal gain, altruism, recommendation from a health professional and encouragement from family and/or friends. For personal gain, although the desire for a cure was a primary motivator, so too was the possibility of obtaining precise insights into their disease status as they perceived clinical trials provided a high standard of care at no further personal expense.

Well the first thing of course is totally for my benefit.
(ID005)
I decided I would apply for a trial, one of the, one
of things I was very keen on, was that...there would
be some thorough scans done, and we would then
have a very good understanding of where my illness
currently sits. (ID014)
It [trial-based care] was just sort of like business
as usual but with perhaps more thorough screening
during the process, that I would have got from my
oncologist because, you know, a lot of these scans
that are being done for example, are expensive, if
you've got to pay for them yourself, and so, that was
on offer. (ID014)

Participating for altruistic purposes was justified using reasons such as assisting in advancing clinical research, enhancing cancer care for fellow cancer survivors or subsequent generations of their family should they be diagnosed with the disease. In the case of the latter, rationalising participation as a positive was associated with encouragement and support from family members:

\begin{abstract}
The second motivation is that if it extends the knowledge of human medicine and condition... if I can do something to, for my future, for my grandchildren I'm happy to do that. I can't see any use in not being around to enjoy them and enjoy the grandchildren and have a happy life. I've got a lot of people who love me and don't want to see me go. (ID005)

Why I'm happy to do the trials because we, firstly we feel people have done this for me, to get me this far down and now it's my turn to step up and be there for the next lot of people who have got the fight in front of them. (ID011)
\end{abstract}

\section{Being randomised}

For most participants, being randomised to a trial treatment was difficult to comprehend and/or accept as allocation to their desired treatment was seen to be left to fate or chance. The effort participants reported 
in getting to the point of deciding to participate in a trial suddenly felt compromised by the uncertainty introduced by potential randomisation to a non-preferred treatment. Additionally, the certainty desired by participants in seeking access to promising new treatments (such as ${ }^{177}$ Lu-PSMA-617) and able to help them live longer or with fewer symptoms in trials felt at risk when confronted with the uncertainty of randomisation.

Participants were eager to receive the new experimental treatment ${ }^{177} \mathrm{Lu}$-PSMA-617. Due to cancer progression after previous treatment, limited treatment options and feeling a sense of hope from trial information, many participants were very optimistic about the prospect of receiving a good outcome from ${ }^{177}$ Lu-PSMA-617 (despite not knowing whether they would receive it). The perceived benefits and odds of receiving the experimental treatment seemed to outweigh the burden of the randomisation process. For some, their desperation to receive ${ }^{177} \mathrm{Lu}-\mathrm{PSMA}$ extended to the point where they were willing to leave the trial and pay for the experimental treatment outside of the trial if not allocated to that treatment arm. This included obtaining a second mortgage, using their superannuation funds, and organising financial support from family and friends.

\begin{abstract}
So that, and then she [health professional] said to me this is on but its random, you've got 50\% chance of getting into the trial and I hoped and hoped. (ID010) I mean, I guess if worse came to worse, we'd probably hock the house and if someone said, 'look, you'll get 5 years extra out of Lutetium', I guess we'd be prepared to hock the house to do that, I guess. (ID006)

There are no strings you could pull or anything to get it, so you've just got to wait for the dice to roll. (ID010)
\end{abstract}

\section{DISCUSSION}

Our findings show the experience of men enrolling in TheraP trial is represented by the four themes of: 'hoping to survive'; 'needing to feel informed'; 'choosing to participate' and 'being randomised' with results suggesting men seeking to enrol in clinical trials of new prostate cancer treatments would benefit from improved informational and decision support.

First, we observed an unusual phenomenon in this study with all participants expressing a negative view at the prospect of receiving further chemotherapy and a strong preference for, or favourable perception of, ${ }^{177}$ Lu-PSMA-617 despite it being a new, unproven experimental treatment. This view is particularly remarkable given cabazitaxel is a proven treatment for men with advanced prostate cancer, ${ }^{29}$ although anticipatory fears about chemotherapy are previously reported in the literature. ${ }^{30}$ Additionally, among men who were randomly assigned to cabazitaxel in the TheraP trial, $14(14 \%)$ withdrew owing to clinical preference and 1 (1\%) withdrew owing to clinical preference and $1(1 \%)$ man met an exclusion criterion (thrombocytopenia) after initial eligibility.

There are several explanations for such strong positive views towards the experimental arm therapy including that ${ }^{177} \mathrm{Lu}$-PSMA treatment was already available as a 'fee-for-service' from private providers where usually, unapproved therapies would not be accessible outside a clinical trial. ${ }^{31}$ Moreover, there were published data and publicly available experiences shared on popular survivorship forums prior to the TheraP trial commencement..$^{3-33}$ It is also possible that given 12 men participating in this study had already received docetaxel chemotherapy, emphatic expressions of hope around the 'new' treatment may have emanated from both awareness of published data and experiences of others receiving ${ }^{177} \mathrm{Lu}$-PSMA, and a desire to avoid further chemotherapy treatment and its side effects.

The availability of Lu-PSMA as a fee for service and the peer-to-peer sharing of treatment experiences on survivorship forums has implications for clinicians and researchers working with an increasingly digitally literate population of prostate cancer survivors. ${ }^{34}$ With limitations in the quality of peer-to-peer anecdotal evidence shared online and inaccuracies in internet-based information on prostate cancer treatments ${ }^{35}$ clinical trialists and clinicians should adopt a communication approach to clarify individual patient motivations for joining a trial, provide relevant and personalised trial information to prospective participants and discuss their expectations of outcomes to promote shared decision-making. ${ }^{36-38}$

Men in our study reported the need to feel informed about the trial and a need for more user-friendly, simpler, succinct trial information. A focus for improving the trial experience must include reducing information overload by providing effective trial decision support tools. Recent evidence suggests the 'high stakes' nature of clinical trial information and discussion at recruitment may be psychologically challenging and cognitively complex to individuals, influencing their decision to accept trial participation. ${ }^{39-41}$ Although cancer information overload among survivors is a well-described phenomenon in the literature, overloading an individual with information does not appears to be associated with the decision to enrol in a clinical trial. ${ }^{39}$ However, reducing the feeling of information overload is associated with a positive attitude towards participating in clinical trials with a recent study reporting that simple, personalised and interactive messages improved individuals' attitudes and informed consent as part of the trial and lowered feelings of cancer information overload. ${ }^{42}$

Interventions catering to the communication preferences of participants and promoting opportunities for feedback about the nature of trial participation are necessary, particularly where such interventions promote opportunities for conversations between 
clinicians, trialists and participants. ${ }^{42,} 43$ Although previous review-level evidence shows a lack of effective decision support in the context of clinical trials, ${ }^{44}$ a recent intervention was reported to be effective where information was delivered in a multimodal interactive format to promote shared decision-making. ${ }^{45,46}$

\section{Strengths and limitations}

This study addresses a key gap in the literature in understanding the experiences of men with advanced prostate cancer in a clinical trial. Strengths of this study include being nested within the trial, obtaining perspectives and experiences of deciding whether to participate in a trial postenrolment, but prerandomisation reduced the chance of participants rationalising the result of their randomisation in a positive or negative manner. Limitations of this study are that it does not include patients who ultimately chose not to participate in the trial, additionally that we were unable to interview partners or family of men deciding to enrol to the extent of identifying the role of family in making a decision about whether to participate in a clinical trial.

\section{CONCLUSION}

This study provides an insight into patient experience of a novel treatment for advanced prostate cancer. Our results indicate that participants preferred to be randomised to the experimental arm despite a lack of evidence about effectiveness. Furthermore, we identified that uncertainty about survival, the advanced disease context and the subsequent experience of deciding to enrol in a clinical trial interlinks with the need for information and support surrounding the decision whether to enrol in a clinical trial. For these men, there is a desire for simple, effective and engaging trial information allowing them to judge whether a clinical trial may offer the chance to achieve their treatment-related goals. Further research is required to implement interventions that promote informed consent and shared decision-making. Please see online supplemental file 2 for references 16-46.

\footnotetext{
Author affiliations

${ }^{1}$ School of Nursing and Midwifery, University of Southern Queensland, Toowoomba, Queensland, Australia

${ }^{2}$ Prostate Cancer Theranostics and Imaging Centre of Excellence (ProsTIC), Molecular Imaging and Therapeutic Nuclear Medicine, Peter MacCallum Cancer Centre, Melbourne, Victoria, Australia

${ }^{3}$ Sir Peter MacCallum Department of Medicine, The University of Melbourne, Melbourne, Victoria, Australia

${ }^{4}$ Faculty of Health, University of Technology Sydney, Sydney, New South Wales, Australia

${ }^{5}$ Faculty of Health Sciences, Australian Catholic University—Brisbane Campus, Brisbane, Queensland, Australia

${ }^{6}$ Centre for Health Research, University of Southern Queensland, Toowoomba, Queensland, Australia

${ }^{7}$ Prostate Cancer Foundation of Australia, St Leonards, New South Wales, Australia
}

${ }^{8}$ Exercise Medicine Research Institute, Edith Cowan University, Perth, Western Australia, Australia

${ }^{9}$ CeMPED, The University of Sydney, Sydney, New South Wales, Australia

${ }^{10}$ Department of Medical Oncology, Monash University, Clayton, Victoria, Australia

${ }^{11}$ Department of Medical Oncology, Eastern Health, St. John's, Newfoundland, Canada

Twitter Haryana Dhillon @hagsie

Acknowledgements The authors would like to thank all participants involved in this study. The QualTheraP substudy was completed in partnership with the Australian and New Zealand Urogenital and Prostate (ANZUP) Cancer Trials Group and Prostate Cancer Foundation of Australia (PCFA). We would also like to thank the Peter MacCallum Cancer Centre and Royal Brisbane and Women's Hospital for their assistance to the study team. ANZUP acknowledges funding from Cancer Australia through its 'Support for Cancer Clinical Trials' programme.

Contributors NR and SKC are joint principal investigators and conceived the study. NR and MSH curated the data. BV and NR performed the formal analysis. All authors acquired funding. NR and SKC designed the methodology. BV and NR managed and coordinated the project. NR procured resources to carry out the study. NR, SKC and JD provided oversight and leadership of the project. BV and NR prepared coding frameworks. BV and NR wrote the first draft of the manuscript. All the authors wrote (reviewed and edited) the manuscript. The corresponding author attests that all listed authors meet authorship criteria and that no others meeting the criteria have been omitted.

Funding Funding support was received from a ANZUP Below the Belt Grant (QualTheraP: a nested, multiperspective longitudinal qualitative study of participants). The National Health and Medical Research Council (NHMRC) Centre of Research Excellence provided further funding support through a PhD Scholarship. IDD is supported by an NHMRC Practitioner Fellowship (APP1102604). The TheraP study received support from the Australian Nuclear Science and Technology Organization (ANSTO), Endocyte Inc., a Novartis Company, Movember, The Distinguished Gentleman's Ride, It's a Bloke Thing and CAN4CANCER. MSH is supported by grants from the Prostate Cancer Foundation (PCF) and the Peter MacCallum Foundation. IDD is supported by an NHMRC Practitioner Fellowship (No. 1102604).

Competing interests Authors report no direct relevant competing interests. MSH reports grants from Movember, Prostate Cancer Foundation of Australia, Endocyte (a Novartis Company), Peter MacCallum Foundation and non-financial support from ANSTO, during the conduct of the study; grants from Prostate Cancer Foundation, Movember, Prostate Cancer Foundation of Australia, US Department of Defence; personal fees from Janssen, Sanofi Genzyme, Mundipharma, Astellas, Merck/MSD, outside the submitted work. IDD reports grants from National Health and Medical Research Council, during the conduct of the study; institutional payments to support prostate cancer trials from Pfizer, ANZUP Cancer Trials Group, Bayer, Astellas, Janssen, Movember Foundation, Merck Sharp \& Dohme outside the submitted work; unremunerated chair of ANZUP Cancer Trials Group. Other authors declare there are no competing interests.

Patient consent for publication Not required.

Ethics approval Ethical approval of the QualTheraP substudy was obtained from the National Health and Medical Research Council (NHMRC) Human Research Ethics Committee (HREC/48397/PMCC-2018). 
Provenance and peer review Not commissioned; externally peer reviewed.

Data availability statement All data relevant to the study are included in the article or uploaded as supplementary information. Not applicable.

\section{ORCID iDs}

Bianca Viljoen http://orcid.org/0000-0003-3412-2607

Haryana Dhillon http://orcid.org/0000-0003-4039-5169

\section{REFERENCES}

1 Culp MB, Soerjomataram I, Efstathiou JA, et al. Recent global patterns in prostate cancer incidence and mortality rates. Eur Urol 2020;77:38-52.

2 Globocan. Prostate. Prostate fact sheet, 2019. Available: https://gco.iarc.fr/today/data/factsheets/cancers/27-Prostatefact-sheet.pdf [Accessed 10 Oct 2020].

3 Ralph N, Chambers S, Pomery A, et al. Nurse-led supportive care intervention for men with advanced prostate cancer. Oncol Nurs Forum 2019;46:92-103.

4 Zajdlewicz L, Hyde MK, Lepore SJ, et al. Health-related quality of life after the diagnosis of locally advanced or advanced prostate cancer: a longitudinal study. Cancer Nurs 2017;40:412-9.

5 Crawford ED, Petrylak D, Sartor O. Navigating the evolving therapeutic landscape in advanced prostate cancer. Urol Oncol 2017;35S:S1-13.

6 James ND, de Bono JS, Spears MR, et al. Abiraterone for prostate cancer not previously treated with hormone therapy. N Engl J Med Overseas Ed 2017;377:338-51.
7 Fizazi K, Tran N, Fein L, et al. Abiraterone plus prednisone in metastatic, castration-sensitive prostate cancer. N Engl J Med Overseas Ed 2017;377:352-60.

8 Gilbert DC, Duong T, Kynaston HG, et al. Quality-of-life outcomes from the prostate adenocarcinoma: transcutaneous hormones (PATCH) trial evaluating luteinising hormonereleasing hormone agonists versus transdermal oestradiol for androgen suppression in advanced prostate cancer. BJU Int 2017;119:667-75.

9 Umstead KL, Kalia SS, Madeo AC, et al. Social comparisons and quality of life following a prostate cancer diagnosis. J Psychosoc Oncol 2018;36:350-63.

10 Ralph N, Ng SK, Zajdlewicz L, et al. Ten-year quality of life outcomes in men with prostate cancer. Psychooncology 2020;29:444-9.

11 ClinicalTrials.gov. Clinical trials trends, charts and maps, 2020. Available: https://clinicaltrials.gov/ct2/resources/trends

12 Comis RL, Miller JD, Aldigé CR, et al. Public attitudes toward participation in cancer clinical trials. J Clin Oncol 2003;21:830-5.

13 Unger JM, Cook E, Tai E, et al. The role of clinical trial participation in cancer research: barriers, evidence, and strategies. Am Soc Clin Oncol Educ Book 2016;35:185-98.

14 Gerido LH, He Z. Improving patient participation in cancer clinical trials: a qualitative analysis of HSRProj \& RePORTER. Stud Health Technol Inform 2019;264:1925.

15 Spittler CA. Exploration of how women make treatment decisions after a breast cancer diagnosis. 172. Ann Arbor: University of Kansas, 2011. 